\title{
Chapter 11. Treatment of hypertension under special conditions
}

\author{
Hypertension Research (2009) 32, 70-77; doi:10.1038/hr.2008.12
}

\section{POINT 11A}

White coat hypertension

1. White coat hypertension is observed in $15-30 \%$ of hypertensive patients, and its frequency increases in elderly people.

2. The risk of white coat hypertension developing into sustained hypertension and leading to cardiovascular events appears to be high.

3. White coat hypertension is defined as an average clinic blood pressure $>140 / 90 \mathrm{~mm} \mathrm{Hg}$ and an average home blood pressure $\leqslant 135 / 85 \mathrm{~mm} \mathrm{Hg}$ or an average 24-h blood pressure on ambulatory blood pressure monitoring (ABPM) $\leqslant 130 /$ $80 \mathrm{~mm} \mathrm{Hg}$.

4. White coat hypertension basically requires lifestyle modifications and close follow-up, but the administration of antihypertensive drugs should also be considered if the risk of organ damage or cardiovascular events is high.

\section{Masked hypertension}

1. Masked hypertension is observed in $10-15 \%$ of the normotensive general population and about $30 \%$ of treated hypertensive patients with apparently well-controlled clinic blood pressure of $\leqslant 140 / 90 \mathrm{~mm} \mathrm{Hg}$.

2. Cardiovascular risk is two to three times higher compared with that at a normal blood pressure and is comparable with that in sustained hypertension.

3. A diagnosis of masked hypertension is made when the average clinic blood pressure is $\leqslant 140 / 90 \mathrm{~mm} \mathrm{Hg}$ and home blood pressure is $\geqslant 135 / 80 \mathrm{~mm} \mathrm{Hg}$ or the average 24-h blood pressure on ABPM is $\geqslant 130 / 80 \mathrm{~mm} \mathrm{Hg}$.

4. Masked hypertension includes morning hypertension, stressinduced hypertension, such as workplace hypertension, and nocturnal hypertension.

5. In antihypertensive treatment based on the morning blood pressure, it should be controlled at $\leqslant 135 / 85 \mathrm{~mm} \mathrm{Hg}$.

\section{1) HYPERTENSION BASED ON DIURNAL CHANGES IN BLOOD PRESSURE}

Clinic blood pressure is not necessarily in agreement with the outof-clinic blood pressures measured during daily activities such as those measured at home or on ABPM. Blood pressure can be classified into normotension, white coat hypertension, masked hypertension and sustained hypertension according to clinic and out-of-clinic blood pressures (Figure 11-1). ${ }^{608}$

a. White coat hypertension

White coat hypertension is a condition in which blood pressure measured in clinic is hypertensive but that measured out of the clinic is normal in untreated patients (Figure 11-1). ${ }^{608}$ White coat hypertension is defined as an average clinic blood pressure of $\geqslant 140 / 90 \mathrm{~mm} \mathrm{Hg}$ and an average home blood pressure or daytime blood pressure on ABPM of $<135 / 85 \mathrm{~mm} \mathrm{Hg}$ or an average 24-h blood pressure of $<130 / 80 \mathrm{~mm} \mathrm{Hg}$ on multiple measurements.

White coat hypertension is observed in $15-30 \%$ of patients diagnosed with hypertension by a clinic blood pressure of $\geqslant 140$ / $90 \mathrm{~mm} \mathrm{Hg}$, and its frequency increases in elderly people. ${ }^{608}$ Organ damage is mild, and cardiovascular prognosis is good in white coat hypertension compared with sustained hypertension. ${ }^{79,149,608}$ However, it is controversial whether the outcome of white coat hypertension is comparable with that of normotension. The white coat phenomenon is not necessarily a benign condition and may be related to an increase in blood pressure under intense stress. White coat hypertension may develop into sustained hypertension in the future and may be a risk factor for cardiovascular events in the long term. ${ }^{83,84,89,609}$ These risks are higher in individuals in whom the out-of-clinic systolic and diastolic pressures are in the high-normal range, being $125-135$ and $80-85 \mathrm{~mm} \mathrm{Hg}$, respectively, and in those with other cardiovascular risk factors, such as obesity and metabolic syndrome, or organ damage, such as microalbuminuria. ${ }^{609}$ Therefore, the evaluation of other risk factors and organ damage is necessary in treating white coat hypertension.

Basically, white coat hypertension should be treated by lifestyle modifications without drug treatment. Patients should be examined periodically, attention should be paid to stressful situations in daily lives, and changes in lifestyle and self-measurement of home blood pressure should be encouraged. Antihypertensive medication may be required in patients with high-risk white coat hypertension in whom out-of-clinic blood pressure is relatively high or is complicated by cardiovascular disease, organ damage, diabetes mellitus or metabolic syndrome.

\section{b. Masked hypertension}

Masked hypertension is a condition in which clinic blood pressure is normal but out-of-clinic blood pressure is hypertensive (Figure 11-1). ${ }^{250,608}$ Masked hypertension is defined as an average 


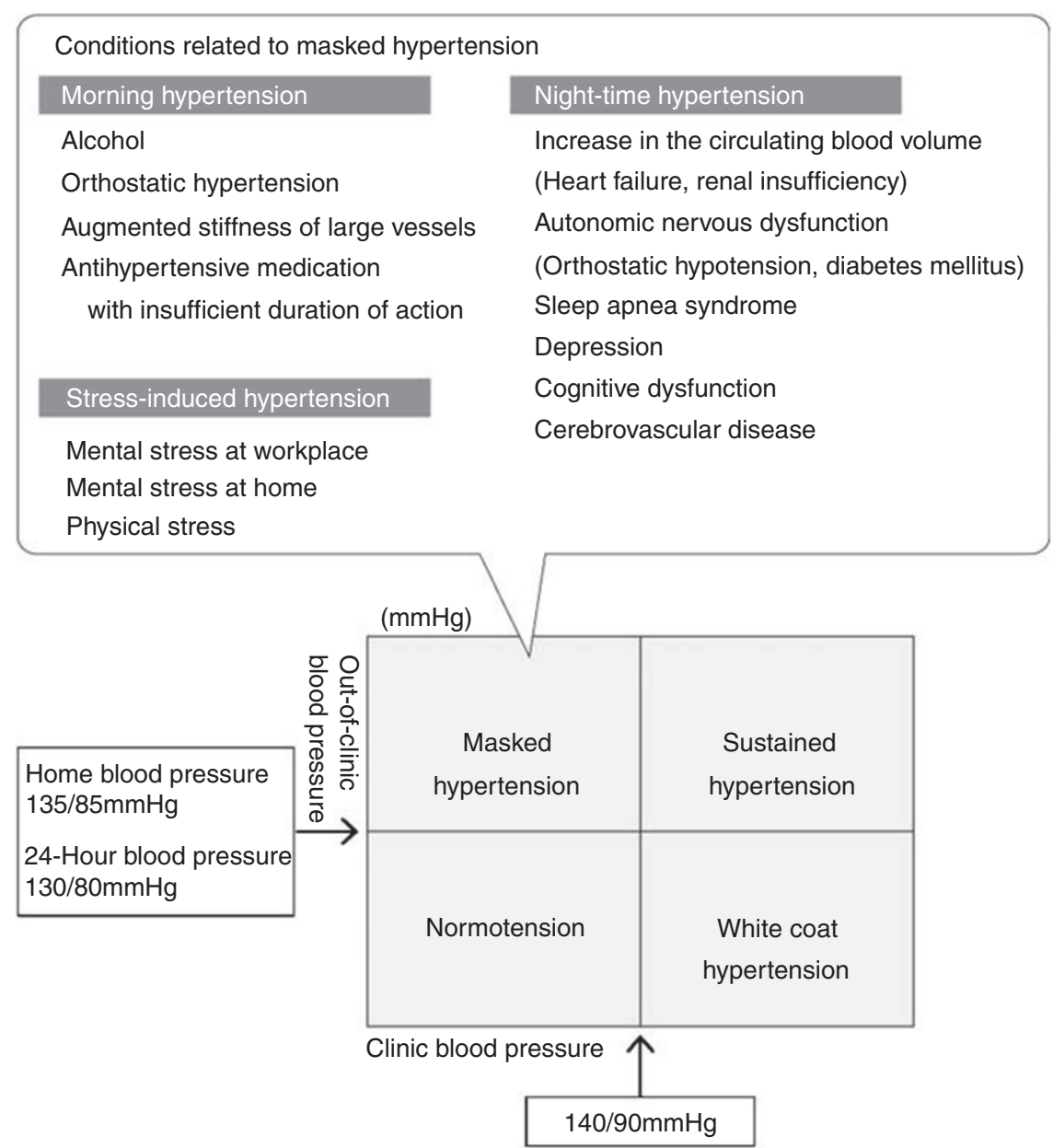

Figure 11-1 Diagnosis of white coat and masked hypertension.

clinic blood pressure on multiple measurements of $<140 / 90 \mathrm{~mm} \mathrm{Hg}$ and an average daytime blood pressure on multiple measurements at home or on ABPM of $\geqslant 135 / 85 \mathrm{~mm} \mathrm{Hg}$ or an average 24 -h blood pressure of $\geqslant 130 / 80 \mathrm{~mm} \mathrm{Hg}$.

Masked hypertension, which is defined by the clinic and out-ofclinic blood pressures, exhibits diverse pathological features. Morning, workplace and nocturnal hypertension constitutes masked hypertension. ${ }^{610}$ These hypertensive states are distinguished by the period of increase in the out-of-clinic blood pressure.

Masked hypertension is observed in $10-15 \%$ of the general population with normotension and about $30 \%$ of hypertensive patients in whom blood pressure is adequately controlled by antihypertensive treatment to $<140 / 90 \mathrm{~mm} \mathrm{Hg} .{ }^{250,611}$ In masked hypertension, the risks of organ damage and cardiovascular events are significantly higher than in normotension or white coat hypertension and are comparable with those in sustained hypertension. Previous clinical studies have shown that masked hypertension is more often accompanied by metabolic abnormalities than normotension and is associated with advanced hypertensive organ damage, such as left ventricular hypertrophy and carotid artery thickening, whether the patient is treated or untreated. ${ }^{612,613}$ The relative risk of cardiovascular disease in masked hypertension was two to three times higher than that in normotension and was comparable with that in sustained hypertension in follow-up studies of the general population and hypertensive patients undergoing treatment. ${ }^{88,89,250,614}$
The diagnosis and treatment of masked hypertension should be started with the measurement of home blood pressure. All hypertensive patients under antihypertensive treatment, patients with a high-normal blood pressure (130-139/85-89 mm Hg), smokers, heavy drinkers, people under intense mental stress (workplace, home), those with a high physical activity level, those with a high heart rate, those with abnormal orthostatic blood pressure changes (orthostatic hypertension, orthostatic hypotension), those with obesity, metabolic syndrome or diabetes mellitus and those with organ damage (particularly left ventricular hypertrophy) or cardiovascular disease constitute a high-risk group for masked hypertension (Table 11-1). ${ }^{299,615,616}$ It is important to measure home blood pressure or ABPM in these high-risk individuals regardless of clinic blood pressure.

Points regarding antihypertensive treatment for masked hypertension are to control blood pressure in the normal range over $24 \mathrm{~h}$, to perform antihypertensive treatment on the basis of early morning blood pressure and to maintain it at $<135 / 85 \mathrm{~mm} \mathrm{Hg}$.

\section{c. Morning hypertension}

Although there is no consensus on the definition of morning hypertension, a condition in which blood pressure is specifically higher in the morning than at other times of day may be regarded as morning hypertension in a narrow sense. As the criterion of hypertension based on home blood pressure is $135 / 85 \mathrm{~mm} \mathrm{Hg}$, an 
Table 11-1 High-risk groups suspected to have masked hypertension

- Hypertensive patients with antihypertensive treatment

- People with a high-normal blood pressure (130-139/85-89 mm Hg)

- Smokers, heavy drinkers

- People with a high mental stress level (workplace, home)

- People with a high physical activity level

- People with a high heart rate

- People with abnormal orthostatic changes in the blood pressure (orthostatic hypertension, orthostatic hypotension)

- Patients with obesity, metabolic syndrome or diabetes mellitus

- Patients with advanced target organ damage (particularly left ventricular hypertrophy, increased intima-media thickness of carotid artery)

- Patients with cardiovascular disease

average blood pressure early in the morning of $\geqslant 135 / 85 \mathrm{~mm} \mathrm{Hg}$ is defined as morning hypertension in a broad sense.

Cardiovascular events frequently occur early in the morning, and blood pressure increases from the nighttime to early in the morning due to diurnal changes. As early morning blood pressure is significantly associated with the risk of brain, heart and kidney damage and all cardiovascular risks, morning hypertension, in which the blood pressure is increased in the time of the highest cardiovascular risk, is important. ${ }^{93,610}$ Furthermore, the problem is that the greatest decrease in antihypertensive effect is attenuated early in the morning despite adequate control of clinic blood pressure in hypertensive patients on antihypertensive treatment.

Morning hypertension is associated with two types of circadian blood pressure variation. One is an extension of nocturnal hypertension. The other is the surge type, a rapid increase in blood pressure before and after waking up. Both types of morning hypertension are considered to be possible risk factors for cardiovascular disease. The greatest augmentation of variability in autonomic nervous activities and blood pressure in relation to reduced baroreceptor reflex is observed in the period from midnight to early morning. ${ }^{617}$ Regardless of the 24-h blood pressure level, a high blood pressure level early in the morning, increased variability of the blood pressure and morning surge, that is, a rapid increase in blood pressure from midnight to early morning, are risk factors for cardiovascular events ${ }^{86,91,618}$ and organ damage, such as left ventricular hypertrophy, carotid arteriosclerosis and silent cerebral infarction. ${ }^{86,618-621}$ Moreover, the risk of cardiovascular events is considered to be enhanced early in the morning, as various risk factors-including increased platelet aggregation and thrombophilic tendencies, as well as increased activities of the sympathetic nervous system and neuroendocrine systems, such as the renin-angiotensin-aldosterone system (RAAS) - act additively or synergistically to exacerbate organ damage.

Target organ damage is more advanced in morning hypertension than in hypertension defined on the basis of clinic blood pressure, ${ }^{622}$ and follow-up studies have reported that the risk of stroke $e^{93,94}$ and a loss of functional independence in those aged 75 years or above is high. $^{57}$

Factors involved in the morning surge include mental stress, habitual drinking and the severity of obstructive apnea syndrome in addition to cold exposure and age. ${ }^{623}$ Therefore, appropriate heating of the house in the morning in winter, moderate drinking and good quality of sleep are suggested to be important as nondrug treatments of morning hypertension.

The addition of antihypertensive treatment targeted at controlling morning hypertension to conventional treatment is expected to facilitate complete control of blood pressure over $24 \mathrm{~h}$, including at night, and allows more effective prevention of cardiovascular events. The target of blood pressure control should be $<135 / 85 \mathrm{~mm} \mathrm{Hg}$ early in the morning, but control at an even lower level $(<130 / 80 \mathrm{~mm} \mathrm{Hg}$ over $24 \mathrm{~h}$ ) is desirable in high-risk hypertensive patients with diabetes or chronic kidney disease.

In morning hypertension, long-acting antihypertensive drugs, the actions of which continue over $24 \mathrm{~h}$, should be used. Even when prescribing a once-daily long-acting antihypertensive drug, modifications such as splitting the dose into morning and evening are necessary if a high morning blood pressure is observed. In addition to the bedtime dosage of a $\mathrm{Ca}$ channel blocker, the administration of sympatholytic drugs ( $\alpha$-blockers, a centrally acting sympatholytic drug) and renin-angiotensin (RA) system inhibitors (angiotensin-converting enzyme (ACE) inhibitors and angiotensin receptor blockers (ARBs)) before going to bed suppresses neurohumoral factors (the activities of which are enhanced early in the morning), significantly reduces morning blood pressure and protects target organs. Strict control of morning hypertension is usually difficult with a single antihypertensive drug, and combination therapy is often required. However, if the dose of an antihypertensive drug is increased to lower peak blood pressure at a particular time of day, symptoms such as malaise and dizziness may be exacerbated at other times due to an excessive fall in blood pressure. Timed antihypertensive therapy, in which different classes of antihypertensive drugs are administered at different times of day, may be necessary.

\section{d. Nocturnal hypertension}

Nocturnal hypertension is defined as an average nighttime blood pressure on ABPM of $\geqslant 120 / 70 \mathrm{~mm} \mathrm{Hg}$. In addition, the riser type, in which blood pressure is higher during the night than during the day, increases cardiovascular risk and is regarded as nocturnal hypertension in a narrow sense. Measurement of nighttime blood pressure and evaluation of abnormal diurnal changes in blood pressure such as in the riser type are performed by ABPM, which has been covered by medical insurance since April 2008. Recently, it has become possible to automatically measure blood pressure during sleep using a home blood pressure-measurement device. ${ }^{95}$

Nighttime blood pressure is less variable than daytime blood pressure and is more closely related to cardiovascular risk or cognitive function. ${ }^{102,624}$ Blood pressure decreases by $10-20 \%$ during the nighttime compared with daytime blood pressure (normal diurnal changes, dipper) in normal diurnal blood pressure change. The risks of organ damage to the brain, heart and kidney, cardiovascular events and cardiovascular mortality are high in the nondipper type in which blunted nocturnal fall in blood pressure and in the riser type in which blood pressure increases during the nighttime. ${ }^{85,335,610}$ It is detected as 'morning hypertension' on home blood pressure measurement when a high nighttime blood pressure persists even after awakening. Elevated nocturnal blood pressure promotes vascular damage and exaggerates cardiovascular risk, even though home blood pressure level is normal in the early morning and before going to bed. ${ }^{625,626}$

Nocturnal hypertension is a consequence of sleep disorders such as sleep apnea syndrome, an increase in circulating blood volume due to heart failure and renal insufficiency and disorders of the autonomic nervous system, such as those in diabetes mellitus, particularly orthostatic hypotension. Depression, impairment of cognitive function, brain atrophy and cerebrovascular disorders are also related to the nondipper type and riser type of nocturnal hypertension. 
Restriction of salt intake and diuretics are useful for the control of nocturnal hypertension. ${ }^{627}$ Furthermore, the administration of antihypertensive drugs before sleep that are targeted at controlling morning hypertension is recommended. There has been a report that a nondipper could be converted to a dipper type, and better 24-h blood pressure control could be achieved by changing the timing of administration of one of the drugs to before going to bed without increasing the number of drugs used. ${ }^{628}$

\section{e. Stress-induced hypertension}

A condition in which clinic blood pressure is normal but the average blood pressure in the daytime, during which the patient is exposed to stress at the workplace or home, consistently exceeds a criterion of $135 / 85 \mathrm{~mm} \mathrm{Hg}$ for example, is regarded as stress-induced hypertension.

Mental and physical stress is known to affect the blood pressure measured by ABPM. ${ }^{629}$ Workplace hypertension is a variation of stress-induced hypertension. Workplace hypertension, defined as blood pressure being normal on health screening or in the clinic but increased at the workplace under stress, is associated with obesity or a family history of hypertension. ${ }^{90}$ ABPM or blood pressure measurement at the workplace is necessary to detect stress-induced hypertension, which could be missed by blood pressure measurement in the clinic or home blood pressure measurement in the morning and before sleep. Although evidence with regard to its clinical significance and its diagnosis and treatment is still insufficient, careful follow-up is necessary.

In shift workers, diurnal changes in blood pressure are dependent on the individual's sleeping pattern rather than daytime or nighttime hours. Therefore, antihypertensive drugs that are effective when administered once a day should be taken just after waking in the evening if the patient sleeps during the daytime. However, as sympathetic activities do not decrease sufficiently during daytime compared with nighttime sleep, a dip in blood pressure during sleep is less likely to occur, leading to a high frequency of nondipper-type abnormality in diurnal blood pressure changes in shift workers. ${ }^{630}$

\section{POINT 11B}

Hypertensive emergencies and urgencies

1. In patients suspected of having a hypertensive emergency, the diagnosis and evaluation of the pathological condition must be performed by prompt examination, and treatment must be initiated without delay.

2. As acute target organ damage progresses in hypertension, complicating hypertensive encephalopathy or acute aortic dissection, hypertensive left ventricular failure accompanied by pulmonary edema, acute coronary syndrome accompanied by severe hypertension, pheochromocytoma crisis, eclampsia and so on, the patients must be admitted, and i.v. antihypertensive treatment must be started immediately. In principle, treatment should be conducted in facilities with a hypertension specialist. The same approach should be taken for accelerated-malignant hypertension.

3. Sustained marked hypertension (usually $\geqslant 180 / 120 \mathrm{~mm} \mathrm{Hg}$ ) not accompanied by the progression of acute organ damage is regarded as a hypertensive urgency, and control of blood pressure is attempted by oral medication. However, as many such patients have organ damage or show resistance to treatment, they should be referred to a hypertension specialist.

\section{2) DIAGNOSIS AND TREATMENT OF HYPERTENSIVE EMERGENCIES AND URGENCIES}

\section{a. Definition and classification}

A hypertensive emergency is not simply an abnormally high blood pressure but is a condition in which acute damage has occurred and is progressing in target organs such as the brain, heart, kidney and large vessels due to marked hypertension (usually $\geqslant 180 / 120 \mathrm{~mm} \mathrm{Hg}$ ). It must be diagnosed promptly, and antihypertensive treatment must be started immediately. Hypertensive emergencies include hypertensive encephalopathy, hypertension complicated by acute aortic dissection, hypertensive left ventricular failure accompanied by pulmonary edema, acute coronary syndrome accompanied by marked hypertension, pheochromocytoma crisis, eclampsia and so on (Table 11-2). ${ }^{631,632}$ Marked hypertension with no rapid progression of organ damage is regarded as a hypertensive urgency. In these cases, there is no evidence of improvement in the outcome by emergency antihypertensive treatment. Whether or not the condition is an emergency should not be judged according to blood pressure alone. Emergency antihypertensive treatment is not indicated even with an abnormally high blood pressure if there is no acute or progressive organ damage, but it is necessary in hypertensive encephalopathy due to eclampsia or acute glomerulonephritis and aortic dissection even when blood pressure is not abnormally high. The pathological condition must be clarified promptly (Table 11-3), the judgment of whether or not it is an emergency case must be made, and which drugs should be used, how they should be administered, what the target blood pressure level should be and how soon it could be attained must be determined. However, the initiation of treatment for emergencies must not be delayed by spending time on thorough evaluation.

\section{b. Principles for treatment}

Hypertensive emergencies must, in principle, be treated by hospitalization. Intravenous antihypertensive treatment should be performed in an intensive care unit or a similar environment. Invasive monitoring of blood pressure is desirable. Due to the presence of organ damage and vascular lesions, an unnecessarily rapid and excessive decrease in blood pressure is most likely to induce ischemic events, such as brain infarction, cortical amaurosis, myocardial infarction and progression of renal dysfunction due to a decrease in organ perfusion pressure. Therefore, the use of drugs and administration methods that allow the degree and rate of decrease in blood pressure to be predicted and the effects to be promptly adjusted is desirable. General targets of blood pressure control are a decrease in mean blood pressure of no more than $25 \%$ during the first $1 \mathrm{~h}$ and to a level of $160 / 100-110 \mathrm{~mm} \mathrm{Hg}$ within the next $2-6$ h. ${ }^{38}$ However, in patients with aortic dissection, acute myocardial infarction, acute coronary syndrome and hypertensive encephalopathy with no preceding hypertension (acute glomerulonephritis, eclampsia and so on), blood pressure level at which treatment should be initiated and target control level should be set lower.

Once the initial target of blood pressure control has been reached, and oral medication is started, i.v. treatment is gradually reduced until discontinuation. Although few parenteral drugs for antihypertensive treatment are available in Japan, they are shown in Table 11-4, with their dosage/regimens, time needed to act and duration of actions, adverse effects, points requiring caution and special indications. With nitroprusside, the rate and degree of decrease in blood pressure are easy to adjust because of the rapid onset and short duration of its action. Cyanate poisoning is unlikely to occur at doses $<2 \mu \mathrm{g} \mathrm{kg}^{-1} \mathrm{~min}^{-1}$. In Japan, however, due to the 
Table 11-2 Hypertensive emergencies

- Accelerated-malignant hypertension with papilledema

- Hypertensive encephalopathy

- Severe hypertension ${ }^{a}$ associated with acute organ damage Atherothrombotic brain infarction

Brain hemorrhage

Subarachnoid hemorrhage

Head trauma

Acute aortic dissection

Acute left ventricular failure

Acute coronary syndrome

Acute or rapidly progressive renal failure

- Severe hypertension ${ }^{\mathrm{a}}$ after thrombolytic therapy for brain infarction

- Excess circulating catecholamines

Pheochromocytoma crisis

Interactions of monoamine oxidase inhibitors with foods or drugs

Use of sympathomimetic drugs

Rebound hypertension after sudden cessation of antihypertensive drugs

Automatic hyperreflexia after spinal cord injury

- Eclampsia

- Hypertensive emergencies related to surgery

Severe hypertension ${ }^{\mathrm{a}}$ in patients requiring emergency surgery

Postoperative hypertension

Postoperative bleeding from vascular suture lines

- Hypertension after coronary bypass surgery

- Severe body burns

- Severe epistaxis

Accelerated-malignant hypertension, perioperative hypertension, rebound hypertension, burn and epistaxis are classified as urgencies if they are not severe.

Prepared on the basis of refs. 702 and 703 .

a'Severe hypertension' here is not in agreement with hypertension categories of the JSH2OO4

Guidelines. The blood pressure requiring emergency antihypertensive treatment should be

evaluated in each condition. Prepared on the basis of Kaplan ${ }^{631}$ and Rosei et al. ${ }^{632}$

lack of experience with the use of nitroprusside and its adverse effects, Ca channel blockers have been more frequently used. Caution is necessary in dose adjustment with $\mathrm{Ca}$ channel blockers because of their relatively long duration of effect.

In patients with hypertensive urgency, a long history of hypertension and chronic organ damage is often observed. This suggests that the lower limit of autoregulation of organ blood flow is high. Although antihypertensive treatment should be started within a few hours after diagnosis, blood pressure should be reduced relatively slowly to about $160 / 100 \mathrm{~mm} \mathrm{Hg}$ over $24-48 \mathrm{~h}$ thereafter. Many hypertensive urgencies can be controlled by oral medication. Oral administration of the capsule contents of nifedipine or bolus i.v. injection of the Ca channel blocker, nicardipine, should be avoided as it can cause an excessive decrease in blood pressure and reflex tachycardia. Ca channel blockers with a relatively rapid onset of action (short-acting or intermediate-type $\mathrm{Ca}$ channel blockers), ACE inhibitors, the $\alpha \beta$-blocker, labetalol, or $\beta$-blockers should be administered orally, or loop diuretics may be used concomitantly depending on the condition. Dose adjustment is easy with captopril because of the rapid onset and relatively short duration of its action, but it should be started at a low dose $(6.25-12.5 \mathrm{mg})$ as it may cause an excessive decrease in blood pressure in malignant hypertension and in a dehydrated state, in which activities of the RA system are enhanced. Caution is necessary in patients with renal dysfunction because hyperkalemia is most likely to occur 1-2 days after commencing treatment with ACE inhibitors. ACE inhibitors should not be used in patients suggested to have bilateral renovascular hypertension or renovascular hypertension with a functionally solitary kidney, as they may cause renal failure. If they
Table 11-3 Check items for evaluating conditions suspected to be hypertensive emergencies

History, symptoms

History of diagnosis and treatment of hypertension, state of use of sympathomimetic drugs and other drugs

Headache, visual impairment, neurological symptoms, nausea/vomiting, chest/

back pain, cardiac/respiratory symptoms, oliguira, body weight changes and so on Physical findings

Blood pressure: repeat measurements (diastolic pressure is often $\geqslant 120 \mathrm{~mm} \mathrm{Hg}$ ),

laterality and so on

Pulse, respiration, body temperature

Evaluation of the body fluid volume: tachycardia, dehydration, edema, measure-

ment of the blood pressure in the standing position and so on

Central nervous system: disturbance of consciousness, convulsion, hemiparesis and so on

Ocular fundus: linear or flame-shaped hemorrhage, soft exudate, retinal edema, papilledema and so on

Neck: jugular vein distension, bruit and so on

Chest: cardiac enlargement, heart murmur, sings of heart failure and so on

Abdomen: hepatomegaly, bruit, (pulsatile) mass and so on

Limbs: edema, arterial pulsation and so on

Emergency examinations

Urinalysis, blood cell count (including smears)

Blood chemistry (urea nitrogen, creatinine, electrolytes, glucose, LDH, CPK and so on)

ECG, chest X-rays, arterial blood gas analysis as indicated

Cardiac and abdominal ultrasonography, brain CT scan or MRI, chest/abdominal CT scan, as indicated

Blood sampling for the measurement of the plasma renin activity, aldosterone, catecholamine and BNP concentrations, as indicated

Abbreviations: BNP, brain natriuretic peptide; $\mathrm{CPK}$, creatine phosphokinase

ECG, electrocardiogram; LDH, lactate dehydrogenase.

Intravenous injection of a low dose of phentolamine if pheochromocytoma is suspected.

are used, monitoring of the serum creatinine and potassium levels is necessary. In patients with hypertensive urgencies, initial treatment is possible at the outpatient clinic, but careful observation in the facility for 5-6 h after the beginning of administration and on an outpatient basis for 2-3 days thereafter with adjustment of the regimen is necessary. Then, the blood pressure should be controlled to the final target primarily using long-acting antihypertensive drugs, and antihypertensive treatment should be continued. However, treatment by hospitalization is also desirable for a hypertensive urgency in high-risk patients, such as those with a history of cardiovascular disease.

\section{c. Hypertensive encephalopathy}

Hypertensive encephalopathy is a condition in which a rapid or marked increase in blood pressure disrupts the autoregulation of cerebral blood flow, causing brain edema due to an unnecessarily high blood flow and pressure. It is most likely to occur when blood pressure rises to $\geqslant 220 / 110 \mathrm{~mm} \mathrm{Hg}$ in chronically hypertensive patients and to $\geqslant 160 / 100 \mathrm{~mm} \mathrm{Hg}$ in normotensive individuals. ${ }^{633}$ Some patients show no proteinuria or hypertensive retinopathy. Hypertensive encephalopathy is the most severe hypertensive emergency leading to brain hemorrhage, disturbance of consciousness, coma and death without appropriate treatment. It is accompanied by exacerbating headache, nausea/vomiting, disturbance of consciousness, convulsions and so on, but focal symptoms are relatively rare. As emergency reduction of blood pressure must, in principle, be avoided in stroke, its exclusion is important. On MRI, vascular edema is often observed in the white matter of the parietal to occipital lobes. 
Table 11-4 Parenteral drugs for the treatment of hypertensive emergencies

\begin{tabular}{|c|c|c|c|c|c|}
\hline Drug & Dosage/regime & $\begin{array}{l}\text { Onset of } \\
\text { action }\end{array}$ & $\begin{array}{l}\text { Duration } \\
\text { of action }\end{array}$ & Adverse effects/points requiring caution & Special indications \\
\hline \multicolumn{6}{|l|}{ Vasodilators } \\
\hline Nicardipine & $\begin{array}{l}\text { i.v. infusion } \\
0.5-6 \mu \mathrm{ggg}^{-1} \mathrm{~min}^{-1}\end{array}$ & $5-10 \mathrm{~min}$ & $15-30 \mathrm{~min}$ & $\begin{array}{l}\text { Tachycardia, headache, flushing, } \\
\text { local phlebitis and so on }\end{array}$ & $\begin{array}{l}\text { Most emergencies. Caution with high } \\
\text { intracranial pressure or acute coronary } \\
\text { syndrome }\end{array}$ \\
\hline Diltiazem & $\begin{array}{l}\text { i.v. infusion } \\
5-15 \mu \mathrm{kg}^{-1} \mathrm{~min}^{-1}\end{array}$ & Within $5 \mathrm{~min}$ & $30 \mathrm{~min}$ & $\begin{array}{l}\text { Bradycardia, AV block, sinus arrest and } \\
\text { so on. A low dose in unstable angina }\end{array}$ & Most emergencies except acute heart failure \\
\hline Nitroglycerin & $\begin{array}{l}\text { i.v. infusion } \\
5-100 \mu \mathrm{g} \mathrm{min}^{-1}\end{array}$ & $2-5 \min$ & $5-10 \mathrm{~min}$ & $\begin{array}{l}\text { Headache, vomiting, tachycardia, } \\
\text { methemoglobinemia, tolerance } \\
\text { with prolonged use and so on. } \\
\text { Must be protected from light }\end{array}$ & Acute coronary syndrome \\
\hline $\begin{array}{l}\text { Sodium } \\
\text { nitroprusside }\end{array}$ & $\begin{array}{l}\text { i.v. infusion } \\
0.25-2 \mu g \mathrm{~kg}^{-1} \mathrm{~min}^{-1}\end{array}$ & Immediate & $1-2 \min$ & $\begin{array}{l}\text { Nausea, vomiting, tachycardia, } \\
\text { cyanate poisoning at a high dose or } \\
\text { prolonged administration and so on. } \\
\text { Must be protected from light }\end{array}$ & $\begin{array}{l}\text { Most emergencies. Caution with high } \\
\text { intracranial pressure or renal dysfunction }\end{array}$ \\
\hline Hydralazine & i.v. injection $10-20 \mathrm{mg}$ & $10-20 \mathrm{~min}$ & $3-6 h$ & $\begin{array}{l}\text { Tachycardia, flushing, headache, } \\
\text { exacerbation of angina pectoris, sustained } \\
\text { hypotension and so on }\end{array}$ & Eclampsia (not the first choice) \\
\hline \multicolumn{6}{|c|}{ Sympatholytic drugs } \\
\hline Phentolamine & $\begin{array}{l}\text { i.v. injection } 1-10 \mathrm{mg} \text {, } \\
\text { infusion at } 0.5-2 \mathrm{mg} \mathrm{min}^{-1} \\
\text { after an initial bolus } \\
\text { injection is also possible }\end{array}$ & $1-2 \min$ & $3-10 \mathrm{~min}$ & Tachycardia, headache and so on & $\begin{array}{l}\text { Pheochromocytoma, excess circulating } \\
\text { catecholamines }\end{array}$ \\
\hline Propranolol & $\begin{array}{l}\text { i.v. injection } 2-10 \mathrm{mg}\left(1 \mathrm{mg} \mathrm{min}^{-1}\right) \rightarrow \\
2-4 \mathrm{mg} \text { every } 4-6 \mathrm{~h}\end{array}$ & & & $\begin{array}{l}\text { Bradycardia, AV block, heart failure } \\
\text { and so on }\end{array}$ & Tachycardia induced by other drugs \\
\hline
\end{tabular}

Abbreviation: $A V$, arteriovenous.

If pulmonary edema, heart failure or body fluid retention is noted, or tolerance has developed, furosemide or carperitide should be used concomitantly.

As the autoregulation of cerebral blood flow is disturbed, a rapid and marked decrease in blood pressure is most likely to cause brain ischemia. Treatment should be started with i.v. preparations (continuous i.v. infusion). The rate of decrease in blood pressure should be adjusted by monitoring blood pressure and neurological symptoms. Treatment should be conducted to achieve an approximately $25 \%$ decrease in blood pressure over the first 2-3 h. Nicardipine, diltiazem and nitroprusside can be used. If an increase in extracellular fluid is observed, or resistance has developed, furosemide should be used concomitantly. Hydralazine, which increases the intracranial pressure, must be avoided.

\section{d. Cerebrovascular disease}

See Section 1 of Chapter 6.

\section{e. Hypertensive acute left ventricular failure}

Treatment for hypertensive left ventricular failure accompanied by pulmonary edema must be started immediately. Nitroprusside, which has a rapid onset of action and alleviates not only afterload, but also preload by dilating the venous system, is desirable, but nicardipine is also useful. Although nitroglycerin has a relatively weak antihypertensive effect, it is useful when hypertensive acute left ventricular failure is concurrent with ischemic heart disease. Furosemide should be used with these drugs to control pulmonary edema. If there is marked pulmonary congestion, carperitide ( $\alpha$-type human atrial natriuretic peptide preparation) should be used simultaneously. ${ }^{634}$ Although no clear target of blood pressure control has been set, blood pressure should be reduced (usually an approximately $10-15 \%$ decrease in systolic blood pressure) by exam- ining the symptomatic improvements. After blood pressure has been reduced to a certain level, treatment should be shifted to oral medication primarily using RA system inhibitors, such as ACE inhibitors and an ARB combined with Ca channel blockers, and so on.

\section{f. Severe hypertension complicating acute coronary syndrome (acute myocardial infarction and unstable angina)}

To treat angina attacks accompanied by an increase in blood pressure, a nitrite preparation should be administered sublingually or sprayed intra-orally first. If hypertension complicates acute coronary syndrome, nitroglycerin should be administered by continuous i.v. infusion to reduce the myocardial oxygen demand and increase the coronary blood flow as well as lower blood pressure. A $\beta$-blocker can be used concomitantly if there is no contraindication such as marked bradycardia. If a $\beta$-blocker cannot be used, or blood pressure cannot be reduced sufficiently, diltiazem should be used. The administration of $\beta$-blockers and ACE inhibitors from an early stage of myocardial infarction is reported to be useful for improving the outcome.

\section{g. Aortic dissection}

See Section 4 of Chapter 6.

\section{h. Pheochromocytoma crisis}

Pheochromocytoma crisis means a rapid increase in blood pressure due to an excessive secretion of catecholamines. Phentolamine should be administered at $2-5 \mathrm{mg}$ every $5 \mathrm{~min}$ until blood pressure is stabilized. After i.v. injection of the first dose, phentolamine may be 
administered by continuous i.v. infusion. Oral medication using drugs such as the selective $\alpha$-blocker doxazosin should be started simultaneously. Although $\beta$-blockers are effective for the management of tachycardia, they should be used after the administration of $\alpha$-blockers at a sufficient dose. Pheochromocytoma may cause hypertensive encephalopathy, acute left ventricular failure or accelerated malignant hypertension, and treatment mainly using an $\alpha$-blocker should also be performed in such situations.

\section{i. Accelerated-malignant hypertension}

In accelerated-malignant hypertension, diastolic blood pressure is $\geqslant 120-130 \mathrm{~mm} \mathrm{Hg}$, and renal dysfunction progresses rapidly. If left untreated, the general condition rapidly deteriorates and cardiovascular complications, including heart failure, hypertensive encephalopathy and brain hemorrhage, occur, leading to a poor outcome. Its pathological characteristics are fibrinoid necrosis and proliferative intimitis following arteriolar endothelial damage and the infiltration of plasma components into the vascular wall due to prolonged, marked hypertension; pathological findings in the kidney are called malignant nephrosclerosis. In this condition, a vicious cycle of progressive renal dysfunction and an increase in blood pressure is established. Ophthalmoscopic findings include retinal hemorrhages, soft exudates, retinal edema and/or papilledema. In the brain, the autoregulation of blood flow is disrupted by vascular damage, and if brain edema occurs, hypertensive encephalopathy may result. When there was no effective antihypertensive treatment, the outcome of this pathological condition was as poor as that of malignant neoplasm, so it was called malignant hypertension. Malignant hypertension accompanied by papilledema (grade IV according to the Keith-Wagener classification) and accelerated hypertension accompanied by retinal hemorrhages and exudative lesions (grade III) used to be distinguished. However, as there is no difference between them in the progression of organ damage or survival rate, they have recently been combined as accelerated-malignant hypertension. As it is not an independent disease, it is appropriate to call it malignant-phase hypertension. ${ }^{66}$ High blood pressure at the onset of hypertension, interruption of antihypertensive treatment and long-standing mental and physical stress are related to the development of malignant hypertension. ${ }^{635}$ Its incidence has decreased recently due to the spread of antihypertensive treatment, improvements in social and living environments, and so on. According to results from the same facility, organ damage such as ophthalmoscopic findings, left ventricular hypertrophy and renal dysfunction were less advanced recently (1984-1999) than they have been in the past (1971-1983). ${ }^{636,637}$ Not only essential but also secondary hypertension, such as renal parenchymal or renovascular hypertension, may lead to accelerated malignant hypertension.

Although accelerated-malignant hypertension is regarded as an urgency, the condition in which arteriolar lesions progress should be classified as an emergency. ${ }^{66}$ The objective of treatment can often be achieved through oral medication. As many patients have a long history of hypertension, a rapid decrease in blood pressure is associated with the risk of ischemia of important organs. Blood pressure should be reduced to no less than a diastolic pressure of $100-110 \mathrm{~mm} \mathrm{Hg}$ during the first $24 \mathrm{~h} .{ }^{66}$ ACE inhibitors and ARBs are expected to be effective because body fluid is reduced owing to pressure diuresis, and hyperactivity of the RA system is closely related to the pathogenesis in the conditions that resulted from essential hypertension $^{637}$ or the renal crisis of collagen diseases. However, as these drugs may cause an excessive decrease in blood pressure, their administration should be started at a low dose. Loop diuretics should be used if there is sodium/water retention.

\section{POINT 11C}

Transient increases in blood pressure

1. The history of hypertension including blood pressure levels should be inquired, and if a marked temporary increase in blood pressure is not accompanied by progressive organ damage, emergency antihypertensive treatment is unnecessary except in cases of pheochromocytoma.

2. If a marked increase in blood pressure persists, Ca channel blockers or ACE inhibitors with an intermediate duration of action may be administered orally.

3. If sufficient inquiry suggests psychological factors, the patient may be referred to a specialist in mental health care.

\section{Preoperative and postoperative blood pressure management}

1. For the prevention of perioperative complications in hypertensive patients, a differential diagnosis of secondary hypertension, such as pheochromocytoma, and evaluation of hypertensive organ damage and complications, are important.

2. Blood pressure should be controlled by continuous oral or i.v. antihypertensive treatment throughout the perioperative period, including the administration on the morning of surgery.

3. $\beta$-blockers are useful for patients with a high risk of ischemic heart disease.

4. Administration of nifedipine capsules must be avoided because the degree or rate of decrease in blood pressure cannot be controlled.

5. Elimination of pain, anxiety and excitation is also important for controlling the increase in blood pressure.

\section{3) TRANSIENT INCREASES IN BLOOD PRESSURE}

Marked transient increases in blood pressure, except those caused by pheochromocytoma, do not require emergency antihypertensive treatment unless there is progressive or chronic organ damage (Table 11-5). In elderly patients with impairment of the baroreflex mechanism, blood pressure markedly changes and may reach $\geqslant 180-200 / 110-120 \mathrm{~mm}$ Hg. Nifedipine capsules may be administered to such patients, but should be avoided as the resultant rapid and excessive decrease in blood pressure may induce brain or myocardial infarction. Causes of an increase in blood pressure, such as pain and urinary retention, should be removed, and if blood pressure remains high even on repeated measurements, Ca channel blockers or ACE inhibitors with an intermediate duration of action (administered twice a day) may be administered orally.

Although hyperventilation reduces blood pressure in normal individuals, hyperventilation associated with anxiety increases it. ${ }^{638}$ The latter is most likely to occur in hypertensive patients showing nonspecific intolerance to multiple antihypertensive drugs and patients who have experienced panic attacks. Blood pressure can be reduced by treatment for hyperventilation, but some patients may need psychological approaches. Panic disorder is a disease in which patients have repeated episodes of attacks of violent anxiety accompanied by various physical symptoms, including palpitation, dyspnea, chest pain, dizziness, nausea, abdominal pain and so on with a sudden onset (panic attacks), and a paroxysmal increase in blood pressure is observed during attacks due to hyperventilation or an increase in sympathetic tone. Diagnosis and treatment by a specialist are necessary. 
Table 11-5 Conditions that may exhibit marked transient increases in blood pressure

- Impairment of the baroreflex mechanism

- Hyperventilation associated with anxiety

- Panic attacks (panic disorder)

- Pseudopheochromocytoma

- Pheochromocytoma

In 21 patients (among 700 consecutive new hypertensive patients) who exhibited physical symptoms such as paroxysmal headache, chest pain, dizziness, nausea, palpitation, flushing and diaphoresis, with hypertension frequently exceeding $200 / 110 \mathrm{~mm} \mathrm{Hg}$, pheochromocytoma was excluded by laboratory examinations, which revealed only mild elevation of plasma or urinary catecholamines, and imaging studies, and these pathological conditions were diagnosed as 'pseudopheochromocytoma'; 639 consequently, the psychological background is attracting attention. Although overt anxiety or psychological stress related to the attacks is unclear, the involvement of past psychological stress has been suggested. The duration of attacks is $30 \mathrm{~min}$ to several hours, their frequency is one to two times a day to once every two to three months in many patients and blood pressure is normal or mildly elevated during attack-free periods. The condition could be controlled by psychotropic medication and psychotherapy (which was effective in some patients) in addition to the administration of $\alpha \beta$-blockers in 13 patients. These patients are strongly suspected of having pheochromocytoma by internists to whom patients present, but it must be understood that the control of repeated hypertensive attacks is difficult through antihypertensive medication alone and psychotherapeutic approaches are necessary.

\section{4) PREOPERATIVE AND POSTOPERATIVE BLOOD PRESSURE MANAGEMENT}

a. Preoperative evaluation of hypertension

Elective surgery is a good opportunity to assess hypertension and evaluate the therapeutic approach. In patients with untreated hypertension, perioperative risk assessment through the evaluation of hypertensive organ damage and complications, such as those of the brain, heart, kidney, blood vessels and fundus of the eye, as well as a differential diagnosis of secondary hypertension, is important. In particular, it is necessary to examine the presence or absence of conditions in which ischemic complications due to perioperative blood pressure decreases are most likely to occur, such as cerebrovascular disease, carotid artery stenosis, left ventricular hypertrophy, reduced coronary blood flow reserve, ischemic heart disease and renal dysfunction. If there is a risk of ischemic complications, consistent blood pressure management from the preoperative period is necessary to avoid excessive perioperative changes in blood pressure.

In patients suspected of having pheochromocytoma, examinations should be performed by postponing surgery, and if the diagnosis has been established, the tumor must be removed before the intended surgery. Conditions such as renovascular hypertension, primary aldosteronism and Cushing's syndrome pose few problems if blood pressure is controlled to grade I or lower level before surgery, but manageable secondary hypertension should be treated before elective surgery.

Grade I or II hypertension is not an independent risk factor for perioperative cardiovascular complications, but if blood pressure is $\geqslant 180 / 110 \mathrm{~mm} \mathrm{Hg}$ before elective surgery, blood pressure control should precede surgery. ${ }^{640}$ In patients with grade III hypertension, or high-risk hypertension undergoing endoscopic surgery and invasive examinations, the applicability of those procedures must be judged individually by evaluating their risks and merits.

\section{b. Use of antihypertensive drugs in the perioperative period} Antihypertensive drugs should be administered until the day of surgery, in principle, and be resumed as soon as possible after surgery. In particular, if $\beta$-blockers are used, caution is necessary not to interrupt treatment considering the risk of increases in heart rate and blood pressure. $\beta$-blockers protect against perioperative stress and sympathetic hyperactivity and reduce the risk of ischemic cardiac complications and atrial fibrillation. ${ }^{640}$ Therefore, if a patient with ischemic heart disease has not been treated with a $\beta$-blocker, its administration should begin. During surgery, propranolol should be used intravenously if necessary. When using diuretics, the possibility of postoperative dehydration and hypokalemia should be recognized, and if these conditions are manageable, their administration need not be discontinued. If the patient is being treated with an ACE inhibitor or an ARB, it may induce a decrease in blood pressure or renal function associated with a perioperative decrease in blood volume; therefore some reports recommend withholding these drugs on the morning of surgery. ${ }^{640}$ It is necessary to judge individually whether ACE inhibitors or ARBs should be discontinued, particularly in elderly or high-risk patients.

Increases in blood pressure during an emergency or elective surgery should be controlled by the continuous i.v. infusion of Ca channel blockers (nicardipine, diltiazem), nitroglycerin, nitroprusside and so on. As hemodynamics remain unstable after surgery, antihypertensive treatment should be started as early as possible, intravenously if oral administration is impossible. Appropriate treatment is also necessary for factors that increase blood pressure, such as postoperative pain, anxiety and excitation. Administration of nifedipine capsules must be avoided because the degree or rate of blood pressure reduction cannot be controlled.

\section{c. Dental surgery and blood pressure management}

As cardiovascular disease such as stroke may also occur during dental treatment, evaluation of the presence or absence of hypertension and the state of blood pressure control is also necessary before dental treatment. If blood pressure is $\geqslant 180 / 110 \mathrm{~mm} \mathrm{Hg}$, medical consultation and referral should precede dental treatment except for emergency procedures. ${ }^{641}$ Patients receiving antihypertensive medication should be advised to take their medication on the day of dental treatment. Dental procedures that involve pain or anxiety or require a prolonged time induce an increase in blood pressure. ${ }^{642}$ As local anesthetics containing adrenaline (epinephrine) slightly increase blood pressure, their doses should be carefully determined while ensuring that there is sufficient anesthesia for pain control. ${ }^{641,642}$ Prescription of a tranquilizer can be considered in patients complaining of intense anxiety.

\section{Citation Information}

We recommend that any citations to information in the Guidelines are presented in the following format:

The Japanese Society of Hypertension Guidelines for the Management of Hypertension (JSH 2009). Hypertens Res 2009; 32: 3-107.

Please refer to the title page for the full list of authors. 